\title{
Canceling strong and complex interference in NQR-based landmine detection
}

\author{
Weihang Shao*, Panagiotis Kosmas*, Kaspar Althoefer ${ }^{\dagger}$, Jamie Barras* \\ * Department of Informatics \\ King's College London \\ London, UK \\ Email: weihang.shao@kcl.ac.uk \\ ${ }^{\dagger}$ School of Electronic Engineering and Computer Science \\ Queen Mary University of London \\ London, UK
}

\begin{abstract}
Nuclear Quadrupole Resonance (NQR) signal detection is a promising explosives detection technology with applications to humanitarian demining. NQR works in the radiofrequency range, and one challenge to using it in such an application is coping with radiofrequency interference. We herein present an algorithm that cancels strong and complex radiofrequency interference (either stationary or nonstationary) improving the utility of NQR-based landmine detection in humanitarian demining. The algorithm has been tested on both simulated and measured data, and demonstrates good performance in NQR detection, in the presence of strong and complex interference.

Index Terms-Interference cancellation; stationary; nonstationary; NQR signal detection
\end{abstract}

\section{INTRODUCTION}

Landmines and other explosives remnants of conflict cause thousands of casualties and keep millions of acres of land out-of-use long after conflicts end. Current approaches detect anomalies mine-like objects or metal in the ground or use animals (dogs, rats, bees) to sniff for traces of explosives vapour, but it has long been recognized that there is a need in some scenarios (cluttered terrain, ground contaminated with trace explosives from detonations) for an approach that directly detects the presence of bulk explosives.

Nuclear quadrupole resonance (NQR) signal detection has been a useful approach for detecting landmines in recent years [1]-[4]. The explosives can be revealed by transmitting an electromagnetic pulse sequence, as their quadrupolar nuclei resonate and emit the corresponding NQR signal. This technique has high sensitivity and suffers from very few false alarms from misleading objects, because NQR signal has unique features for each explosive material. However, this signal is usually very weak compared to background noise and decays rapidly with time. More seriously, strong and complex interference can easily conceal the NQR signal and is very hard to remove from the acquired data.

An important technique called "echo train" [5], [6] was proposed for promoting NQR data acquisition as well as increasing the signal to noise ratio (SNR). NQR data was traditionally recorded by a single spectrometer. This is not very efficient as for each data acquisition, the spectrometer usually needs a long relax time which is larger than the duration of a fast-decaying NQR signal. By echoing the NQR signal periodically, the "echo train" technique retrieves the NQR signal intensity and initial phase in each echo, and completes the relax time with data of echoes. Besides, the homogeneous initial phase among the echoes suggests that NQR signal in all the echoes of data can be added coherently as opposed to the stochastic noise and interference. Thus the summed data has a higher SNR.

This method however is limited as data collection time is usually prohibitively long in reality, especially in humanitarian demining and security checking [2]. In addition, interference can not be eradicated simply by data summing. So, useful algorithms are needed for proceeding NQR detection after data acquisition. Algorithms based on NQR parameter estimation methods [7], [8] show good performance and robustness on low SNR and weak interference situations. However, the main difficulty of NQR detection lies on canceling strong and complex interference which may be nonstationary and has frequencies which are very close to or even coincide with $\mathrm{NQR}$ frequencies.

Multichannel analysis is a popular method for interference cancelation [9]. In this method, one channel acquires primary data meanwhile the other channels measure interference and noise. However, it is not easy to implement this method in instrumentation or algorithm aspect. Channels may not be isotropic due to calibration errors, and there may be complex phase difference among data acquired by each channel. This method has its limitations in humanitarian demining where equipments/devices must be portable.

Based on single channel premise, we proposed an algorithm which can effectively cancel strong and complex interference. This algorithm estimates the interference components using cost function and approximates the entire interference in acquired data using Fourier basis or wavelets basis. In particular, this algorithm has Fourier basis version for stationary interference and wavelets basis version for nonstationary interference, respectively. The theory of this algorithm has been presented elsewhere in detail [10], [11]. As we know, simple interference, which is stationary or slightly nonstationary and has frequencies which are far from NQR frequencies, can 
be canceled using a frequency selective method [8], [12]. The proposed algorithm can well describe the features of stationary or nonstationary interference, and deal with the overlap between interference and NQR signal, while plain Fourier analysis as well as the related frequency selective methods can not. The proposed interference cancelation (IC) algorithm can be coupled with the well known approximate maximum likelihood (AML) algorithm (based on parameter estimation) [7] facilitating valid NQR detection. This combination is accordingly termed ICAML.

In the next section, we give a summary of ICAML algorithm. In Section III, we assess the performance of ICAML algorithm applied to both simulated data and experimental data. We will summarize the main results in the last section.

\section{SUMmARY OF ICAML ALGORITHM}

In the echo-train system for data acquisition, the NQR signal can be modeled after echo summing [5] as

$$
y(t)=\sum_{k=1}^{d} A_{k} e^{-\frac{\left|t-t_{s p}\right|}{T_{k}^{*}}+j 2 \pi \check{f}_{k} t}
$$

where $t=t_{0}, \ldots, t_{N-1}$ is the $N$ points echo sampling time with the symmetric center to be $t_{s p}, \mu=2 t_{s p}$ is the echo spacing, and $A_{k}, T_{k}^{*}$, and $\check{f}_{k}$ are the amplitude, damping time, and frequency of the $k$ th component, respectively. The raw data in vector form can be written as

$$
\mathbf{Z}_{N}=\mathbf{Y}_{N}+\mathbf{N}_{N}+\mathbf{R}_{N},
$$

where $\mathbf{N}_{N}$ and $\mathbf{R}_{N}$ are the noise and interference parts, respectively. The signal part $\mathbf{Y}_{N}$ satisfies

$$
\mathbf{Y}_{N}=\mathbb{Q}_{N} \mathbf{A},
$$

where $\mathbf{A}$ and $\mathbb{Q}_{N}$ are the amplitude vector and phase matrix respectively, given by,

$$
\mathbf{A}=\left[\begin{array}{llll}
A_{1} & A_{2} & \ldots & A_{d}
\end{array}\right]^{\mathrm{T}},
$$

where $(.)^{\mathrm{T}}$ denotes the transpose, and

$$
\mathbb{Q}_{N}=\left\{a_{I J}\right\}_{(N-1) \times d}=\left\{e^{-\frac{\left|t_{I}-t_{s p}\right|}{T_{J}^{*}}+j 2 \pi \check{f}_{J} t_{I}}\right\}_{(N-1) \times d} .
$$

ICAML algorithm chooses Fourier basis or wavelets basis to precisely approximate stationary or nonstationary interference, respectively [10], [11]. Using cost functions, ICAML is able to determine an optimum basis functions set whose linear combination can effectively model interference. The interference cancelled data $\mathbf{Z}_{N}^{(l)}$ is

$\mathbf{Z}_{N}^{(l)}=\mathbf{Z}_{N}-\left[\begin{array}{llllll}\mathbf{V}_{m_{1}} & \mathbf{V}_{m_{2}} & \ldots & \mathbf{V}_{m_{l}}\end{array}\right]\left[\begin{array}{lllll}\mathbf{V}_{m_{1}} & \mathbf{V}_{m_{2}} & \ldots & \mathbf{V}_{m_{l}}\end{array}\right]^{\dagger} \mathbf{Z}_{N}$

where $\mathbf{V}_{m_{i}}, i=1,2, \ldots, l$ are the selected basis functions, and $(.)^{\dagger}$ denotes the Moore-Penrose pseudo-inverse.

After interference cancellation, ICAML applies the approximate maximum likelihood (AML) method to $\mathbf{Z}_{N}^{(l)}$ to identify the existence of the NQR signal [7]. The amplitudes vector A in Eq. (4) is estimated as

$$
\hat{\mathbf{A}}=\mathbb{Q}_{N}^{\dagger} \mathbf{Z}_{N}^{(l)},
$$

where $(.)^{\dagger}$ denotes the Moore-Penrose pseudo-inverse, yielding the likelihood function

$$
L\left(\check{f}_{k}, T_{k}^{*}\right)=\left[\mathbf{Z}_{N}^{(l)}\right]^{\mathrm{H}} \mathbb{Q}_{N} \mathbb{Q}_{N}^{\dagger} \mathbf{Z}_{N}^{(l)},
$$

where $(.)^{\mathrm{H}}$ denotes the conjugate transpose. Then we can obtain the estimated $\check{f}_{k}$ and $T_{k}^{*}$

$$
\left[\begin{array}{ll}
\hat{\tilde{f}}_{k} & \hat{T}_{k}^{*}
\end{array}\right]=\arg \max _{\tilde{f}_{k}, T_{k}^{*}}(|L|),
$$

where seeking $\check{f}_{k}$ and $T_{k}^{*}$ is respectively among all the possible values of $\check{f}_{k}$ and $T^{*}$ under immediate environment conditions based on knowledge of NQR theory [13]. Hence $\hat{\mathbf{A}}=\mathbb{Q}_{N}^{\dagger}\left(\hat{\tilde{f}}_{k}, \hat{T}_{k}^{*}\right) \mathbf{Z}_{N}^{(l)}$. The acquired $\hat{\tilde{f}}_{k}$ and $\hat{T}_{k}^{*}$ can be substituted into the AML test statistic [14],

$$
T\left(\mathbf{Z}_{N}^{(l)}\right)=(2 N-1) \frac{\left[\mathbf{Z}_{N}^{(l)}\right]^{\mathrm{H}} \mathbb{Q}_{N} \mathbb{Q}_{N}^{\dagger} \mathbf{Z}_{N}^{(l)}}{\left[\mathbf{Z}_{N}^{(l)}\right]^{\mathrm{H}} \mathbf{Z}_{N}^{(l)}-\left[\mathbf{Z}_{N}^{(l)}\right]^{\mathrm{H}} \mathbb{Q}_{N} \mathbb{Q}_{N}^{\dagger} \mathbf{Z}_{N}^{(l)}} .
$$

By predetermining a threshold value $\gamma$, the NQR signal is deemed present if and only if $T\left(\mathbf{Z}_{N}^{(l)}\right)>\gamma$, and otherwise not.

\section{Performance ASSessment of the ICAML ALGORITHM}

This section presents simulated and experimental data to validate the ICAML algorithm. Results are compared to those produced by previously proposed algorithms AML, FSAML [7], [12].

We examines detection of the ${ }^{14} \mathrm{~N}$ NQR signal due to sodium nitrite $\left(\mathrm{NaNO}_{2}\right)$. Under our lab conditions, $\left(\mathrm{NaNO}_{2}\right)$ has only one resonant frequency $\check{f}$ about $1.0365 \mathrm{MHz}$. All possible values of $\check{f}$ is denoted as "NQR band". The frequencies of interference we consider are all centered outside the NQR band. Since the NQR band is very narrow and interference confined only inside the NQR band is very rare and may be filtered in a physical way.

To imitate a landmine, we let a piece of solid $\mathrm{NaNO}_{2}$ suspended in silicone oil served in a sealed plastic box which is buried under soil. The other box without $\mathrm{NaNO}_{2}$ is also prepared for testing probability of false alarms. Data is recorded by a spectrometer with a sampling frequency $f_{s}=\frac{1}{16 \mu \mathrm{s}}$ and sampling points $N=128$ per echo. In the measurements, the frequency band $\left[-\frac{f_{s}}{2}, \frac{f_{s}}{2}\right]$ of the recorded data is modulated from $\left[f_{c}-\frac{f_{s}}{2}, f_{c}+\frac{f_{s}}{2}\right]$, where $f_{c}$ is the frequency center of signal modulation. By setting $f_{c} \simeq \check{f}$, the equivalent NQR frequency is about 0 . 

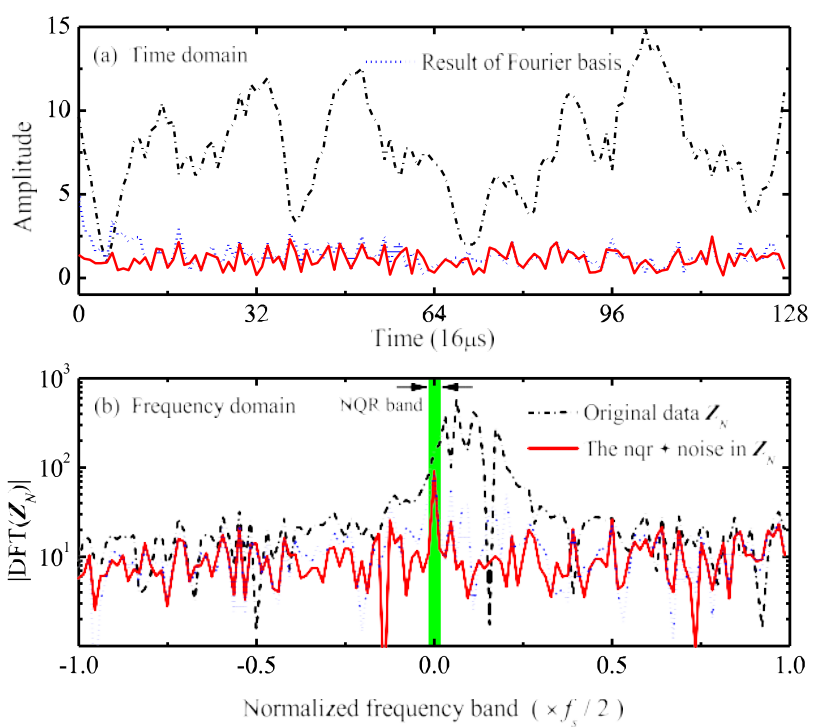

Fig. 1: (Color online) A run of the simulated data (with stationary interference) and its interference canceled form in time domain and frequency domain. "|DFT(.)|" means the absolute value of Discrete Fourier transformation of data. The vertical axis of frequency domain is base-10 logarithmic.

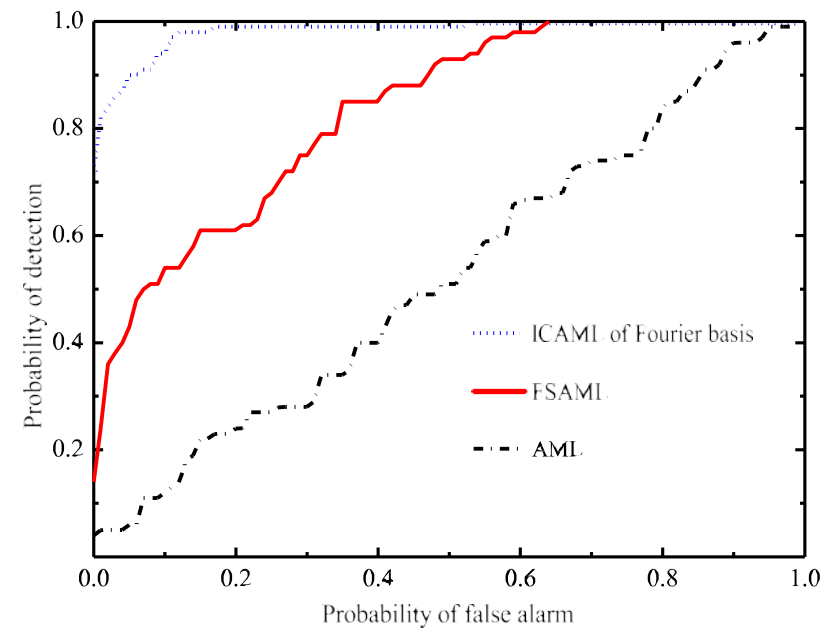

Fig. 2: (Color online) The ROC curves obtained by AML, FSAML, and ICAML algorithms. The results are for the simulated data with stationary interference.

\section{A. Simulated data with stationary interference}

In this subsection, we apply the ICAML algorithm to simulated data that corresponds to the landmine detection problem described above. A simulated data set containing 100 Monte Carlo runs is created, where the NQR signal amplitude is 1 and the noise is circularly symmetric zero-mean Gaussian white noise with variance being 0.25 . Simulated stationary interference is added into this set, which contains multiple frequency components. All these components have their constant frequencies and amplitudes, as well as random initial phases among the 100 runs. In particular, frequencies of some components are very close to the NQR band causing strong overlap between NQR signal and these interference components (see Fig. 1). Since the interference is stationary, the Fourier basis version of the ICAML is better than the wavelets basis version in this case. As shown in Fig. 1, the difference between the original "NQR signal + noise" data and the interference canceled data is very small in both time and frequency domains, which shows that the interference is nicely extracted and removed by ICAML algorithm. Receiver operating characteristic (ROC) curves are shown in Fig. 2. Although the interference can not be handled by the classic AML algorithm, the ICAML algorithm effectively cancels the interference thereby facilitating valid NQR detection. AML's frequency selective (FS) version FSAML alleviates the influence of interference. However, the main limitation of the FS method is that FS can hardly cope with the overlap between the NQR signal and interference [10].

\section{B. Simulated data with nonstationary interference}
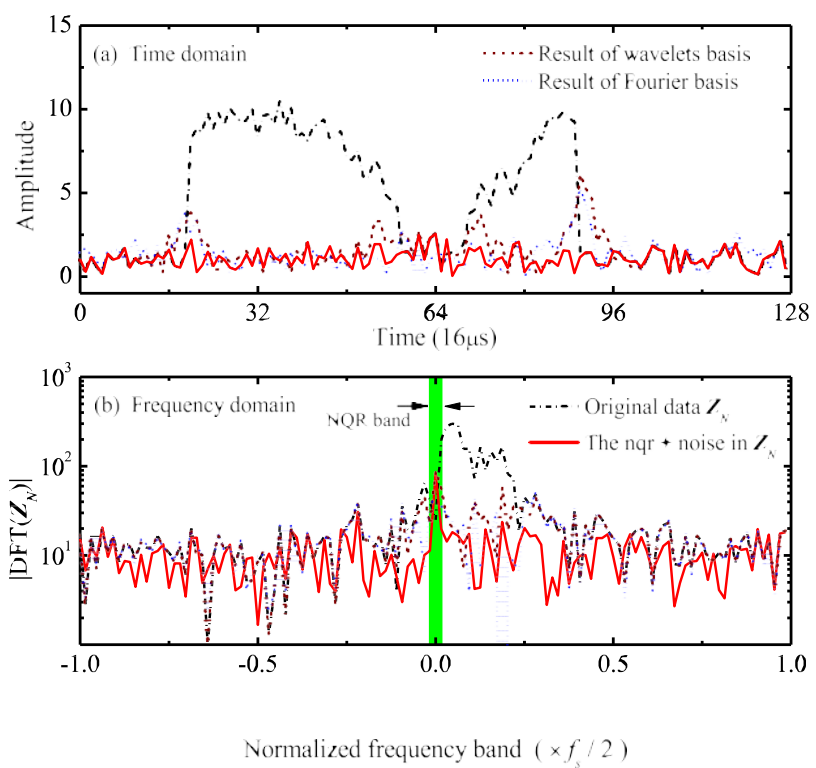

Fig. 3: (Color online) A run of the simulated data (with nonstationary interference) and its interference canceled form in time domain and frequency domain. "|DFT( $\cdot) \mid$ " means the absolute value of Discrete Fourier transformation of data. The vertical axis of frequency domain is base-10 logarithmic.

In this subsection, interference added into the previous simulated data set is nonstationary containing multiple frequency components which all have time-varying frequencies and amplitudes, as well as random initial phases among the 100 runs. In particular, the interference appears intermittently along the time axis, and its spectrum is close to the NQR band causing strong overlap between NQR signal and interference (see Fig. 3). To deal with the time-varying properties of interference, it is necessary to select wavelets basis version of ICAML for interference cancellation. The results in Fig. 3 show that wavelets basis version performs better than Fourier 


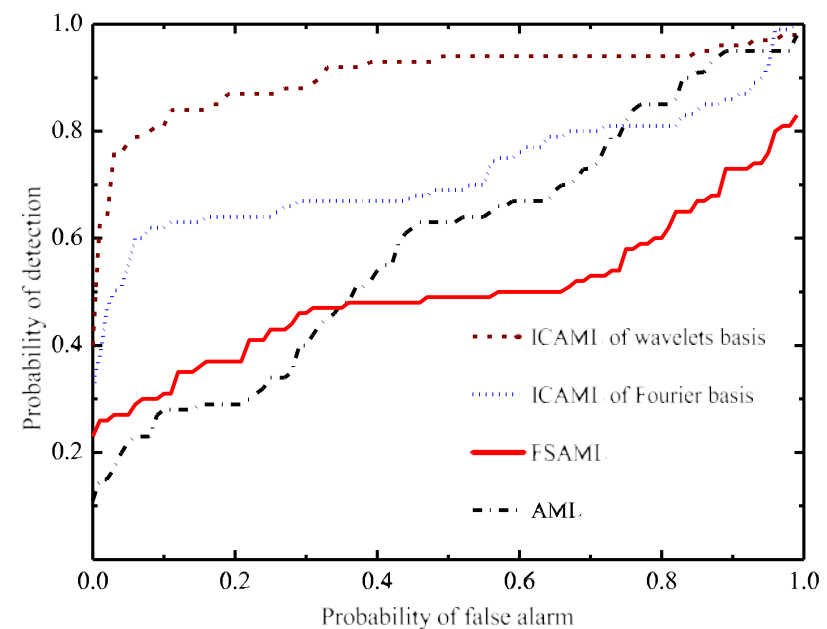

Fig. 4: (Color online) The ROC curves obtained by AML, FSAML, and ICAML algorithms. The results are for the simulated data with nonstationary interference.

basis version in extracting the time-varying interference [11]. This is further confirmed by comparing the ROC curves in Fig. 4, which suggests that wavelets basis is a nice choice for dealing with nonstationary interference when Fourier basis loses performance.

\section{Experimental data test}

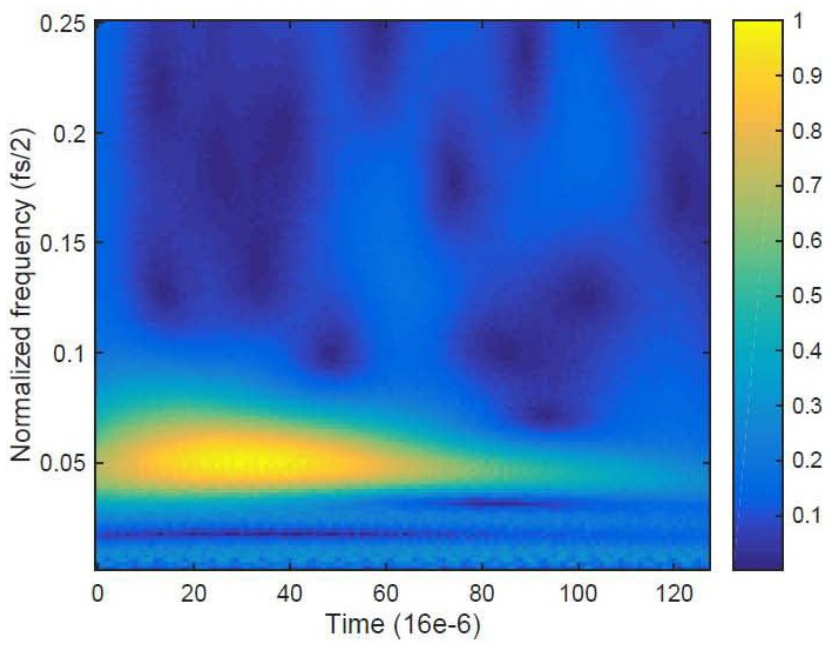

Fig. 5: (Color online) The time-frequency analysis on a run of the experimental data. It is achieved using Morlet wavelets of 'cmor1-1' in Matlab environment. The intensity of the displayed time-frequency information is normalized.

Our experimental data set includes 100 runs. The interference we encountered is nonstationary. A time-frequency analysis on the experimental data (see Fig. 5) confirms the nonstationary properties of interference in the data. For this experimental case, both Fourier basis and wavelets basis can handle the interference well and the ICAML algorithm
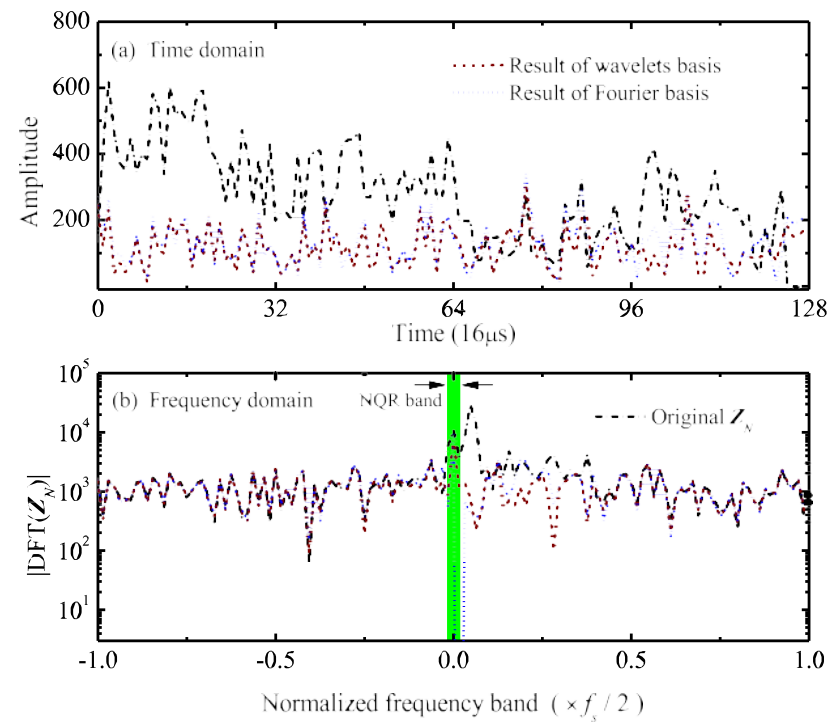

Fig. 6: (Color online) A run of the experimental data and its interference canceled form in time domain and frequency domain. "|DFT(.)|" means the absolute value of Discrete Fourier transformation of data. The vertical axis of frequency domain is base-10 logarithmic.

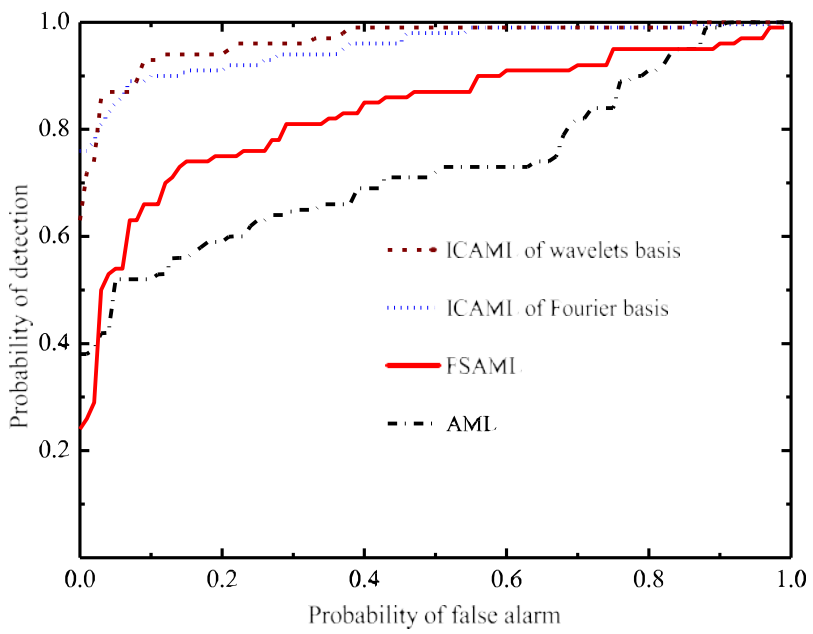

Fig. 7: (Color online) The ROC curves obtained by AML, FSAML, and ICAML algorithms. The results are for the experimental data.

makes good NQR detection (see Figs. 6 and 7). According to our numerical tests, Fourier basis version has robustness on nonstationary interference to some extent. However, wavelets basis version exhibits better performance as it is more suitable than Fourier basis version for dealing with nonstationary interference.

\section{Detecting the NQR signals of TNT}

As TNT is a very common explosive used in landmines, it is worth applying the current algorithm for detecting TNT NQR signals. To test our algorithm for TNT detection, we 

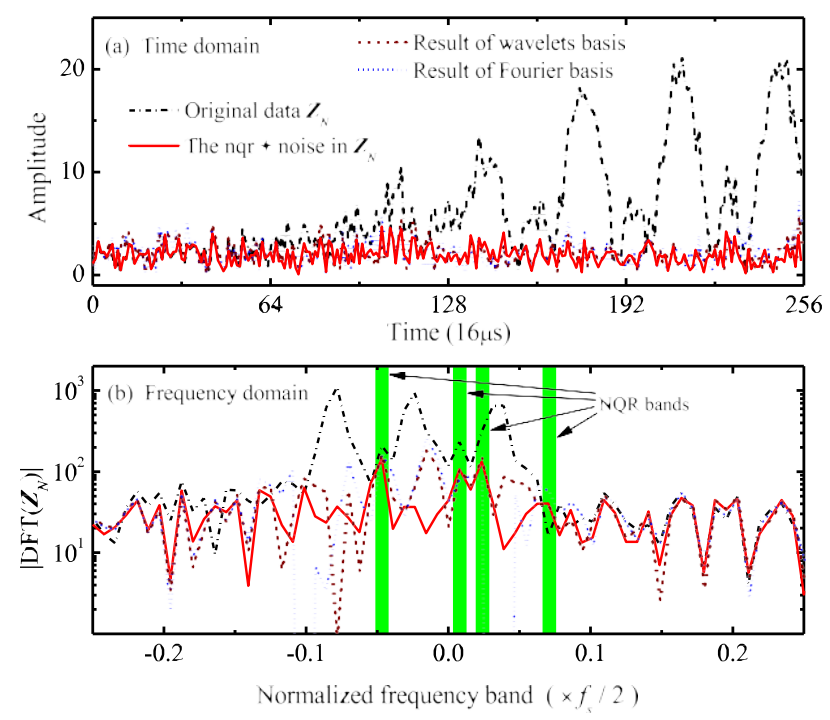

Fig. 8: (Color online) A run of the TNT data and its interference canceled form in time domain and frequency domain. "|DFT( $(\cdot) \mid$ " means the absolute value of Discrete Fourier transformation of data. The vertical axis of frequency domain is base-10 logarithmic.

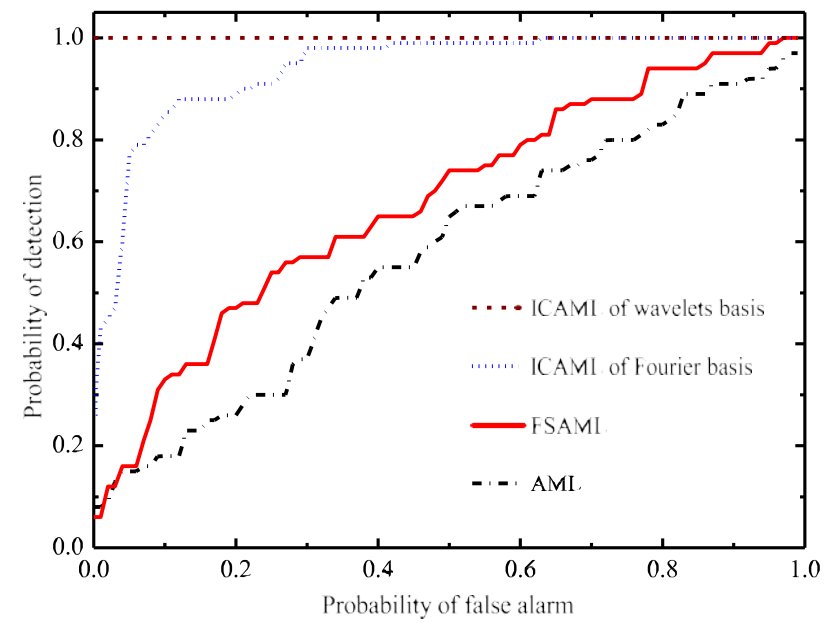

Fig. 9: (Color online) The ROC curves obtained by AML, FSAML, and ICAML algorithms. The results are for the TNT data.

used realistic TNT NQR signal simulations provided by Lund University, and mix them with our simulated stochastic noise and nonstationary interference to create 100 monte carlo data. The interference cancellation results are shown in Fig. (8). As we can see, TNT NQR signal has 4 frequency components. After interference cancellation, the $4 \mathrm{NQR}$ peaks are nicely recovered by wavelet basis version. Figure. 9 shows the almost perfect detection performance of wavelet basis version. Besides, Fourier basis version presents its robustness of detecting NQR signal in this case.

\section{CONCLUSION}

This paper introduces a novel interference cancelation method which can improve NQR signal detection in a background containing strong and complex interference (either stationary or nonstationary). The method can be coupled with the classic NQR detection algorithm approximate maximum likelihood (AML) which assumes the NQR signal is only accompanied with noise, yielding the proposed algorithm ICAML. Both simulated data (including TNT case) and experimental data prove that ICAML algorithm can effectively cancel strong and complex interference and make good NQR detection. Thus ICAML is recommended for real-life demining tasks.

\section{ACKNOWLEDGMENT}

This work has been supported by Find a Better Way (FABW) UK, under Project AQUAREOS. The authors would like to thank Prof. Andreas Jakobsson from Lund University for providing us the simulations of realistic TNT NQR signals.

\section{REFERENCES}

[1] J. A. S. Smith, "Nuclear quadrupole resonance spectroscopy," J. Chem. Educ., vol. 48, no. 1, pp. 39-48, 1971.

[2] N. R. Butt, E. Gudmundson, and A. Jakobsson, "An overview of NQR signal detection algorithms," in Magnetic Resonance Detection of Explosives and Illicit Materials, ser. NATO Science for Peace and Security Series B: Physics and Biophysics, T. Apih, B. Rameev, G. Mozzhukhin, and J. Barras, Eds. Springer, 2013, pp. 19-34.

[3] J. Barras, D. Murnane, K. Althoefer, S. Assi, M. D. Rowe, I. Poplett, G. Kyriakidou, and J. A. S. Smith, "Nitrogen-14 nuclear quadrupole resonance spectroscopy: a promising new analytical methodology for medicines authentication and counterfeit antimalarial analysis," Analytical Chemistry, vol. 84, pp. 2746-2753, 2013.

[4] J. Barras, K. Althoefer, M. D. Rowe, I. Poplett, and J. A. S. Smith, "The emerging field of nuclear qudrupole resonance-based medicines authentication," Appl. Magn. Reson., vol. 43, pp. 511-529, 2012.

[5] M. D. Rowe and J. A. S. Smith, "Mine detection by nuclear quadrupole resonance," in Proc. EUREL Int. Conf. on the Detection of Abandoned Land Mines, pp. 62-66, Oct. 1996.

[6] A. Gregorovič and T. Apih, "Relaxation during spin-lock spin-echo pulse sequence in n 14 nuclear quadrupole resonance," J. Chem. Phys., vol. 129 , p. 214504, 2008.

[7] A. Jakobsson, M. Mossberg, M. D. Rowe, and J. A. S. Smith, "Exploiting temperature dependency in the detection of nqr signals," IEEE Transactions on Signal Processing, vol. 54, no. 5, pp. 1610-1616, 2006.

[8] S. D. Somasundaram, A. Jakobsson, J. A. S. Smith, and K. Althoefer, "Exploiting spin echo decay in the detection of nuclear quadrupole resonance signals," IEEE Trans. Geosc. Remote Sensing, vol. 45, pp. 925-933, 2007.

[9] H. Xiong, J. Li, and G. A. Barrall, "Joint tnt and rdx detection via quadrupole resonance," IEEE Transactions on Aerospace and Electronic Systems, vol. 43, no. 4, pp. 1282-1293, 2007.

[10] W. H. Shao, J. Barras, P. Kosmas, and K. Althoefer, "Detecting nqr signals severely polluted by interference," submitted to Signal Processing.

[11] W. Shao, J. Barras, P. Kosmas, and K. Althoefer, "The use of wavelets basis for cancelling time-varying interference in nqr signal detection," submitted to IEEE Transactions on Signal Processing.

[12] A. Jakobsson, M. Mossberg, M. D. Rowe, and J. A. S. Smith, "Frequency-selective detection of nuclear quadrupole resonance signals," IEEE Trans. Geosc. Remote Sensing, vol. 43, no. 11, pp. 2659-2665, 2005.

[13] S. D. Somasundaram, "Advanced signal processing algorithms based on novel nuclear quadrupole resonance models for the detection of explosives," Ph.D. dissertation, King's College London, 2007.

[14] S. M. Kay, Fundamentals of Statistical Signal Processing, Volume II: Detection Theory. Englewood Cliffs, NJ: Prentice-Hall, 1998. 